\title{
Lesões músculo-esqueléticas relacionadas com as atividades desportivas em crianças e adolescentes: Uma revisão das questões emergentes
}

\author{
Sports related musculoskeletal disorders in children and adolescents: A \\ review of the emerging issues
}

\author{
M.C. Pinho, M.P. Vaz, P.M. Arezes, J.R. Campos, A.B. Magalhães
}

ARTIGO DE REVISÃO | REVIEW ARTICLE

\begin{abstract}
A prática regular de exercício físico promove a melhoria do estado geral de saúde e é fundamental para a prevenção de um grande número de patologias, entre as quais se encontram as doenças músculoesqueléticas. A promoção da atividade física e dos seus efeitos benéficos para a saúde têm contribuído para o rápido crescimento do número de crianças e adolescentes envolvidos em atividades desportivas organizadas ou recreativas que, infelizmente, tem sido acompanhado por um significativo aumento do número de lesões relacionadas com a sua prática. Tendo em conta que o sistema músculo-esquelético das crianças e adolescentes se encontra ainda em desenvolvimento, a sobrecarga associada, em especial, à prática de modalidades desportivas de competição poderá ter reflexos de longo prazo para a sua saúde. Este artigo visa fazer uma revisão bibliográfica sobre os principais aspetos relacionados com as lesões músculo-esqueléticas associadas à prática desportiva das crianças e adolescentes, incluindo os aspetos éticos envolvidos, assim como identificar alguns dos principais fatores de risco e apontar algumas das medidas a adotar para a sua prevenção.

Palavras-chave: lesões músculo-esqueléticas, saúde, crianças e adolescentes, atividades desportivas, educação
\end{abstract}

ABSTRACT

Regular physical activity promotes better general state of health and is essential for the prevention of many illnesses, including musculoskeletal disorders. The promotion of both physical activity and its beneficial effects on health have contributed to the fast growth in the number of children and adolescents involved in organized or recreational sports activities which, unfortunately, has been followed by a significant increase on the number of injuries related to its practice. Taking into account that children and adolescents musculoskeletal system is still developing, its overuse particularly associated to competition sports activities may have long-term effects on their health. This paper aims to review the main questions of sports-related musculoskeletal disorders in children and adolescents, including the involved ethical issues, as well as to identify some of the main risk factors and to point out some of the measures to be adopted for its prevention.

Keywords: musculoskeletal disorders, health, children and adolescents, sports activities, education

Submetido: 09.02.2012 | Aceite: 26.01.2013

Maria Eugénia R. C. Pinho. Universidade do Porto. PROA - LABIOMEP, Porto, Portugal.

Mário A. Pires Vaz, António P. Barbedo de Magalhães. Faculdade de Engenharia da Universidade do Porto, Porto, Portugal.

Pedro M. F. M. Arezes. Escola de Engenharia da Universidade do Minho.

José C. Reis Campos. Faculdade de Medicina Dentária da Universidade do Porto.

Endereço para correspondência: Maria Eugénia R. C. Pinho, Faculdade de Engenharia da Universidade do Porto, Rua Dr. Roberto Frias, Edifício F, Sala 211, 4200-465 Porto, Portugal.

E-mail: epinho@fe.up.pt 
As lesões músculo-esqueléticas (LME) são geralmente definidas como um conjunto de patologias que afetam os músculos, tendões, ligamentos, articulações, nervos, discos vertebrais, cartilagem, vasos sanguíneos ou tecidos moles associados e que podem ser causadas ou agravadas pelas atividades físicas. As LME "abrangem uma vasta gama de doenças inflamatórias e degenerativas do sistema músculo-esquelético" e "são caracterizadas pela dor e pela perda de função física do corpo que limitam as atividades dos indivíduos afetados assim como a sua participação na sociedade" (European Foundation for the Improvement of Living and Working Conditions, 2007, p. 2).

Embora mais comuns entre os idosos, as LME afetam pessoas de todas as faixas etárias (Direcção-Geral de Saúde, 2004; European Bone and Joint Health Strategies Project, 2004), incluindo as crianças e os adolescentes (Foster \& Cabral, 2006).

As LME associadas à prática desportiva podem ser de origem traumática aguda ou resultar da sobrecarga do sistema músculo-esquelético (SME) (Dalton, 1992; Ivkovic, Franic, Bojanic \& Pecina, 2007). As lesões por sobrecarga ou lesões por trauma repetitivo são danos nos tecidos resultantes de movimentos repetitivos acumulados ao longo do tempo (Ivkovic et al., 2007). Entre os jovens atletas a sobrecarga do SME é, cada vez mais, a principal causa de lesões (American College of Sports Medicine, 1993; Brenner \& Council on Sports Medicine Fitness, 2007).

A alteração profunda dos estilos de vida, registada nas últimas décadas nos países desenvolvidos, conduziu a um aumento dramático de patologias como as doenças cardiovasculares, a diabetes, o cancro, a obesidade e as doenças músculo-esqueléticas. Esta situação levou ao reconhecimento internacional da necessidade de promover práticas que contribuam para a inversão dessa tendência e, simultaneamente, permitam minimizar os enormes custos socioeconómicos que elas representam. A impor- tância da prática regular de exercício físico por pessoas de todas as idades é reconhecida pela Organização Mundial de Saúde (OMS) que indica a inatividade física como o quarto fator de risco para a mortalidade global para além de ser uma causa comum de morbilidade (World Health Organization, 2010).

Para as crianças e adolescentes, as atividades desportivas, para além dos efeitos benéficos para a sua saúde, desempenham também um importante papel na sua educação e formação através da transmissão de valores sociais e educativos "como o espírito de equipa, a solidariedade, a tolerância e a competição leal" e contribuem "para desenvolver os conhecimentos, a motivação, as competências e a disponibilidade para fazer esforços pessoais" (Commission of the European Communities, 2007, pp. 2,5; European Commission, 2009).

Contudo, não obstante os inegáveis benefícios da prática de atividades desportivas, o aumento do número de crianças e adolescentes nelas envolvidos tem sido associado a um significativo crescimento do número e da gravidade das lesões desportivas (Aaltonen, Karjalainen, Heinonen, Parkkari \& Kujala, 2007; Adirim \& Cheng, 2003; Brenner \& Council on Sports Medicine Fitness, 2007; Dalton, 1992; Davis, 2004; Ivkovic et al., 2007; Maffulli \& Baxter-Jones, 1995; Micheli \& Klein, 1991), ao mesmo tempo que se tem assistido a uma mudança no padrão das lesões associada à crescente participação em modalidades desportivas organizadas (American College of Sports Medicine, 1993; Brenner \& Council on Sports Medicine Fitness, 2007), com um aumento significativo do peso das lesões por sobrecarga do SME.

Por outro lado, vários autores sustentam a possibilidade deste tipo de lesões, geralmente associadas à especialização numa única modalidade, em idades precoces, poderem ter reflexos de longo prazo na saúde músculo-esquelética dos jovens desportistas (Maffulli, Longo, Gougoulias, Caine \& Denaro, 2010; Maffulli, Longo, Gougoulias, Loppini \& Denaro, 2010; 
Maffulli, Longo, Spiezia \& Denaro, 2010).

Este artigo visa fazer uma revisão bibliográfica sobre os principais aspetos relacionados com as LME associadas à prática desportiva das crianças e adolescentes, incluindo os aspetos éticos envolvidos, assim como identificar alguns dos principais fatores de risco e apontar algumas das medidas a adotar para a sua prevenção.

\section{Identificação e caracterização do problema}

A participação de crianças e adolescentes em atividades físicas tem sido fortemente estimulada a nível mundial com o objetivo de combater os números alarmantes das doenças não comunicáveis que, na sua grande maioria, estão associadas ao estilo de vida dos países desenvolvidos, caracterizado pelo sedentarismo e pelo excesso de peso. Contudo, a crescente participação em atividades desportivas e recreativas tem conduzido a um acréscimo das lesões (Aaltonen et al., 2007; Adirim \& Cheng, 2003). Só nos Estados Unidos, mais de metade dos sete milhões de lesões relacionadas com atividades desportivas ou recreativas que ocorrem todos os anos referem-se a jovens com idades compreendidas entre os 5 e os 24 anos de idade (CDC, n.d.). Alguns dos estudos epidemiológicos referidos na Tabela 1 parecem confirmar a crescente tendência do número de lesões entre os jovens, indicada por diversos autores (Adirim \& Cheng, 2003; American Academy of Orthopaedic Surgeons, 2010; Caine, DiFiori \& Maffulli, 2006; Colvin \& Lynn, 2010; Goldberg, Moroz, Smith \& Ganley, 2007; Gottschalk \& Andrish, 2011; Kerssemakers, Fotiadou, de Jonge, Karantanas \& Maas, 2009; Maffulli \& Baxter-Jones, 1995; Maffulli \& Bruns, 2000; Metzl, 1999; Möller, Åström \& Westlin, 1996; O’Rourke et al., 2007). Por exemplo, Shields e Smith (2006) concluíram que o número de lesões desportivas em crianças dos 5 aos 18 anos que participam em atividades de "cheerleading" mais que duplicou entre 1990 e 2005, ao mesmo tempo que Conn et al. (2004) concluíram que, entre 1997 e 2001, as lesões associadas à prática do "paintball" entre os praticantes com idades iguais ou superiores a 7 anos quase quadruplicaram. Não obstante, um estudo sobre as lesões desportivas entre ginastas com idades compreendidas entre os 6 e os 17 anos, tratadas nos serviços de urgência no período de 1990 a 2005, revelou um decréscimo de $25 \%$ no número de lesões ao longo dos 16 anos de duração do estudo, tendo passado de 28700 em 1990 para 21500 em 2005 (Singh, Smith, Fields \& McKenzie, 2008).

Entre os jovens desportistas as lesões mais frequentes envolvem o SME e representam mais de $80 \%$ das lesões desportivas neste grupo etário (Patel \& Nelson, 2000). Estima-se que só nos Estados Unidos trinta milhões de crianças estejam envolvidas em atividades desportivas organizadas (Adirim \& Cheng, 2003; Demorest \& Landry, 2003), mais de vinte milhões de jovens americanos participem em desportos escolares ou comunitários (University of Michigan, 2009) e que as lesões relacionadas com os desportos, na população dos 5 aos 21 anos, contribuam para cerca de $41 \%$ das LME tratadas nos serviços de urgência (Damore, Metzl, Ramundo, Pan \& van Amerongen, 2003; University of Michigan, 2009). Além disso, cerca de um milhão de jovens americanos todos os anos requerem hospitalização, cirurgia, faltas à escola, ou pelo menos meio-dia em repouso devido a lesões desportivas (University of Michigan, 2009). Na pesquisa efetuada não foram encontrados dados sobre a dimensão do problema em Portugal.

Conforme foi afirmado pelo cirurgião ortopédico americano, especializado em medicina desportiva e Professor Associado de Ortopedia - Dr. DeBerardino - durante o Encontro Anual da Academia Americana dos Cirurgiões Ortopédicos (AAOS) de 2010, "cada vez mais adolescentes participam em atividades desportivas ao longo de todo o ano, sem paragens sazonais, ou participam em múltiplas equipas ao mesmo tempo", de tal modo que as lesões 


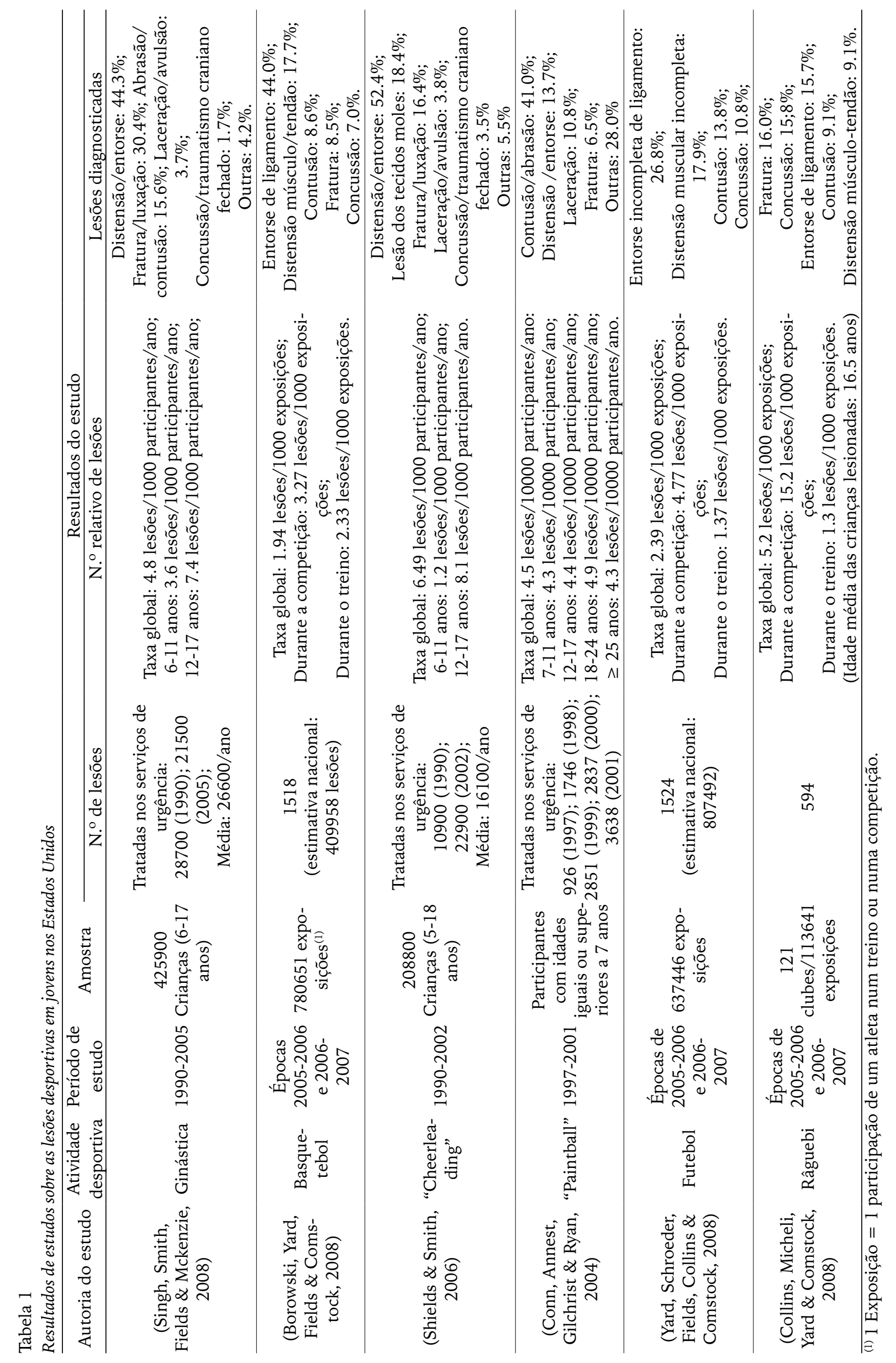


relacionadas com os desportos entre os adolescentes se tornaram uma "epidemia silenciosa" (American Academy of Orthopaedic Surgeons, 2010). De acordo com aquele especialista, "esta exposição crescente indica que continuará a haver um crescimento no número de LME graves, tanto traumáticas como de sobre utilização crónica" e, como os adolescentes não são "adultos em miniatura" e os seus corpos estão ainda em desenvolvimento, as lesões ortopédicas são particularmente preocupantes (American Academy of Orthopaedic Surgeons, 2010). Outros autores evidenciam, igualmente, a importância de ter em conta as características específicas das crianças e dos adolescentes (Davis, 2004; Foster \& Cabral, 2006; Hutchinson \& Nasser, 2000; Stanitski, 1988) e que existem diferenças físicas e fisiológicas que justificam uma maior vulnerabilidade às lesões do que nos adultos (Adirim \& Cheng, 2003; Cirak et al., 2004; Kerssemakers et al., 2009), pelo que as LME são diferentes e se manifestam de forma diferente nos adultos e nas crianças e adolescentes (Caine et al., 2006; Carty, 1998; Côté, Lidor \& Hackfort, 2009; Dalton, 1992; Dohin, 2010; Helms, 1997; Kerssemakers et al., 2009; Malina, 2009; O'Connor \& Groves, 2005; Stanitski, 1997). A maior vulnerabilidade das crianças às lesões músculo-esqueléticas é justificada pelas características anatómicas e biomecânicas, específicas de um sistema músculo-esquelético imaturo (Carty, 1998; Micheli \& Nielson, 2008) ainda em processo de crescimento. Em particular, a ossificação incompleta e a grande quantidade de tecido cartilaginoso contribuem para o aumento da incidência de lesões músculo-esqueléticas por sobrecarga do sistema músculo-esquelético nas crianças e adolescentes (Caine et al., 2006; DiFiori, 2010; Dohin, 2010). Vários autores referem o crescimento acelerado da adolescência como registando uma maior incidência de lesões (Baker, 2003; Purnell, Shirley, Nicholson \& Adams, 2010). Para além da imaturidade do sistema músculo-esquelético, a fase de grande crescimento ósseo (Baker, 2003; Caine et al., 2006; Dalton, 1992; DiFiori, 2010; Kerssemakers et al., 2009; Purnell et al., 2010) da pré-adolescência e da adolescência parece ser ainda mais crítica. Vários autores apontam o desequilíbrio entre a força e a flexibilidade (Kerssemakers et al., 2009) e as mudanças das propriedades biomecânicas do osso dessa fase como a causa da maior incidência de LME (Shanmugam \& Maffulli, 2008). De acordo com Baker (2003) e Baker et al. (2009), durante essa fase, o crescimento dos músculos e tendões não acompanha o elevado ritmo de crescimento ósseo, dando origem a uma maior rigidez nas articulações que, por sua vez, aumenta o risco de lesões por sobrecarga do sistema músculo-esquelético (Baker \& Côté, 2006, p. 97). Por seu lado, o "Committee on Sports Medicine and Fitness" da "American Academy of Pediatrics (AAP)" não recomenda a especialização antes dos 12 ou 13 anos de idade (Callender, 2010) enquanto a OMS aconselha que ela seja evitada antes dos 10 anos de idade (FIMS/WHO Ad Hoc Committee on Sports and Children, 1998). Além disso, os dados da Tabela 1 parecem confirmar uma maior incidência de lesões durante a fase de grande crescimento da adolescência na prática de modalidades como a ginástica e as "cheerleading" por jovens norte americanos, tendo em conta que o número de lesões anuais por mil participantes na faixa etária dos 12 aos 17 anos (ginástica: 7.4; "cheerleading": 8.1) é muito superior àquela que se verifica na faixa etária dos 6 aos 11 anos (ginástica: 3.6; "cheerleading": 1.2) (Shields \& Smith, 2006; Singh et al., 2008) enquanto na prática do "paintball", no que respeita ao número de lesões anuais por 10000 participantes, a faixa etária dos 12 aos 17 anos surge em segundo lugar (4.4) atrás da faixa etária dos 18 aos 24 anos (4.9) (Conn, Annest, Gilchrist \& Ryan, 2004). Essa mesma tendência parece ser confirmada através dos resultados de um inquérito administrado a 73 ginastas acrobáticos com idades compreendidas entre os 8 e os 26 anos 
que mostra as idades dos 11 aos 15 anos como um período crítico para a ocorrência de lesões entre aqueles atletas (Purnell et al., 2010). No mesmo sentido parece apontar um estudo realizado entre jogadores de futebol Australianos que indica os jogadores da faixa etária dos 12 aos 14 anos como aqueles que apresentam o maior número de lesões (Grimmer \& Williams, 2003). Também um estudo sobre as lesões desportivas em crianças dos 5 aos 14 anos de seis países da UE (Áustria, Dinamarca, França, Grécia, Holanda e Reino Unido) mostra que as lesões desportivas (vide Tabela 2) apresentam uma tendência crescente à medida que a idade aumenta e que esse crescimento é significativamente mais elevado nas faixas etárias dos 11-12 anos e dos 13-14 anos (Belechri, Petridou, Kedikoglou, Trichopoulos \& Sports Injuries' European Union group, 2001).

Por outro lado, segundo Hawkins \& Metheny (2001), entre os 5 e os 18 anos, o peso dos rapazes mais que quadruplica e a altura aumenta cerca de $60 \%$ enquanto o peso das raparigas triplica e a altura aumenta cerca de $45 \%$. Durante a fase de crescimento acelerado, os rápidos aumentos da massa e do comprimento dos diversos segmentos corporais envolvidos no movimento vão exigir que uma maior força muscular seja exercida para execução de um dado movimento quando comparada com a força necessária antes dessa fase e que, mesmo que a criança a consiga exercer, vai submeter os tendões e as apófises a grande stress/tensão até que ela se consiga adaptar às novas condições de carga (Hawkins \& Metheny, 2001). As fracturas por avulsão das placas de crescimento, assim como as apofisites são duas das lesões mais características nas crianças e adolescentes nesta fase de crescimento (Benjamin et al., 2002) enquanto as zonas mais vulneráveis a lesões são as placas de crescimento, as superfícies das articulações e os locais das inserções músculo-tendinosas (Helms, 1997). Além disso, devido a diversos aspetos relacionados com o crescimento e desenvolvimento (físico, psicológico, ...) dos adolescentes, os tipos de lesões e a sua gestão são diferentes dos registados nas restantes faixas etárias da população (Patel \& Nelson, 2000).

A gravidade da situação não é, contudo, um exclusivo dos Estados Unidos da América. A tendência crescente das lesões desportivas pediátricas é uma realidade comum a todos os países desenvolvidos, embora o alastramento do estilo de vida ocidental aos países em vias de desenvolvimento, como consequência do aumento do rendimento disponível dessas populações, permita antever a dramática situação que pode estar a germinar nessas regiões sobrepovoadas.

Até então focalizada em patologias como as doenças cardiorespiratórias, as doenças infecciosas, o cancro e a diabetes, a comunidade internacional só no final do século XX, graças à iniciativa do Professor Lars Lidgren e do movimento Bone and Joint Decade, reconhece a necessidade de uma ação global para combater o flagelo das doenças músculo-esqueléticas, que contribua simultaneamente para melhorar a qualidade de vida das pessoas e reduzir os enormes custos socioeconómicos que elas representam para a sociedade (European Bone

Tabela 2

Distribuição (em \%) do número de crianças com lesões desportivas em seis países da EU, em 1998 (baseado em Belechri, et al., 2001)

\begin{tabular}{ccccccc}
\hline Idades & $\begin{array}{c}\text { Áustria } \\
(712)\end{array}$ & $\begin{array}{c}\text { Dinamarca } \\
(4608)\end{array}$ & $\begin{array}{c}\text { França } \\
(959)\end{array}$ & $\begin{array}{c}\text { Grécia } \\
(2034)\end{array}$ & $\begin{array}{c}\text { Holanda } \\
(42000)\end{array}$ & $\begin{array}{c}\text { Reino Unido } \\
(222250)\end{array}$ \\
\hline $5-8$ & 14.89 & 11.39 & 5.74 & 10.13 & 16.31 & 9.79 \\
$9-10$ & 15.73 & 19.88 & 10.01 & 20.11 & 20.86 & 18.05 \\
$11-12$ & 28.09 & 32.40 & 22.42 & 34.46 & 31.05 & 33.06 \\
$13-14$ & 41.43 & 36.35 & 61.73 & 35.30 & 32.10 & 39.09 \\
\hline
\end{tabular}

Nota: entre parêntesis é indicada a dimensão da amostra, na Áustria, Dinamarca e França, e as estimativas nacionais, no caso da Holanda e do Reino Unido. 
and Joint Health Strategies Project, 2004).

O esforço conjunto de organizações como a OMS e o movimento Bone and Joint Decade associado às políticas adotadas na área da saúde pelos governos nacionais tem conduzido a um significativo aumento do número de praticantes de modalidades desportivas. Consequentemente, é cada vez maior o número de pessoas, em particular crianças e adolescentes, que praticam regularmente algum tipo de atividade física. Apesar dos incontestáveis benefícios para a saúde, o rápido crescimento do número de crianças e adolescentes que praticam modalidades desportivas tem-se refletido num acentuado crescimento do número e da gravidade das lesões que lhes estão associadas (Davis, 2004). Paralelamente, registou-se uma significativa mudança nos padrões das atividades físicas desenvolvidas pela maioria das crianças e adolescentes (Anderson, 2005; Johnson, 2008; Lord \& Winell, 2004; Maffulli \& Baxter-Jones, 1995; Maffulli, Longo, Spiezia, et al., 2010; Malina, 2009, 2010). Estes fatores têm contribuído para a alteração da tipologia das LME entre a população mais jovem. Para além das lesões traumáticas agudas (fracturas, entorses, contusões, ...), as lesões desportivas nas crianças e adolescentes são, cada vez mais, motivadas pela sobrecarga repetitiva prolongada a que o SME está sujeito, dando origem a lesões de natureza diferente, tais como fracturas por stress, bursites, tendinites, apofisites das inserções dos tendões e lesões osteocondrais da superfície das articulações (Dohin, 2010; Micheli, Glassman \& Klein, 2010). De acordo com um estudo que analisa as lesões desportivas (394) em crianças na faixa etária dos 5 aos 17 anos, ocorridas entre 1989 e 1991, metade delas eram lesões agudas e $49,5 \%$ eram lesões crónicas e, destas, mais de metade (53\%) afetavam a cartilagem articular e as placas de crescimento das epífises e apófises (Watkins \& Peabody, 1996).

\section{Principais factores de risco das lesões desportivas por sobrecarga}

As lesões desportivas podem ser lesões agudas ou por sobrecarga, sendo que a sobrecarga do SME é responsável por até 50\% da totalidade das lesões observadas na medicina desportiva pediátrica (Dalton, 1992). Anteriormente raras em crianças e adolescentes (American College of Sports Medicine, 1993; Micheli \& Klein, 1991), as lesões por sobrecarga são cada vez mais frequentes (Brenner \& Council on Sports Medicine Fitness, 2007) em consequência dos micro-traumatismos repetitivos durante os treinos (Micheli \& Klein, 1991), provocados pela rotura dos mecanismos de reparação dos tecidos (Ivkovic et al., 2007).

Embora vários autores considerem ser reduzida a probabilidade da sua ocorrência, admitem que as LME por sobrecarga, em crianças e adolescentes, podem provocar a modificação permanente do crescimento ósseo e causar doença e incapacidade prolongadas (Anderson et al., 2000; Buckley, 1994; Helms, 1997; Maffulli, Longo, Spiezia, et al., 2010).

\section{A especialização precoce}

Alguns autores consideram que a escolha de uma única modalidade desportiva ocorre cada vez mais cedo na vida de um número crescente de crianças e adolescentes, submetendo o SME ainda em desenvolvimento a lesões por sobrecarga (DiFiori, 2010; Helms, 1997).

No que respeita ao modelo de desenvolvimento da participação desportiva podem destacar-se duas correntes de pensamento. Por um lado, a teoria da prática deliberada atribuída a Ericsson et al. (1993) que defende a necessidade de envolvimento em formas específicas de prática desportiva, ou seja, a especialização precoce numa única modalidade desportiva de modo a alcançar a máxima proficiência (Baker, Côté \& Deakin, 2005). Ericsson et al. (1993) definem prática deliberada como "uma atividade altamente estruturada que requer esforço, não gera recompensas imediatas, e é moti- 
vada pelo objetivo de melhorar o desempenho ao invés de proporcionar prazer" (Côté et al., 2009). Embora a especialização precoce, caracterizada por uma intensa prática deliberada e um reduzido volume de atividades lúdicas (Côté et al., 2009) ou com exclusão completa de quaisquer outras atividades desportivas ou recreativas, em idades muito jovens (Wiersma, 2000), seja muitas vezes associada ao elevado desempenho dos atletas, é-lhe igualmente atribuída uma variedade de consequências negativas ao nível do desenvolvimento físico, psicológico e social das crianças (Baker, 2003; Baker, Cobley \& Fraser-Thomas, 2009). Para além das lesões desportivas por sobrecarga do SME, uma das consequências nefastas da especialização precoce parece ser o abandono da prática desportiva que vários autores atribuem à falta de divertimento ou prazer sentido pelos jovens atletas na sua prática (Baker et al., 2009; Wall \& Côté, 2007).

A especialização precoce é considerada por alguns autores como sendo a opção prematura - com 6 ou 7 anos de idade (Côté et al., 2009), ou mesmo menos (Callender, 2010) - pela prática de uma única modalidade desportiva.

Face aos efeitos nefastos atribuídos à especialização precoce, vários investigadores propõem um modelo alternativo designado por "diversificação precoce" (Baker et al., 2009; Côté et al., 2009), a que associam, entre outros, a redução do número de lesões por sobrecarga do SME e o importante contributo para "o desenvolvimento da motivação intrínseca e a transferência de competências" (Baker et al., 2009), embora Baker et al. (2009) não tenham encontrado evidências adequadas que suportem qualquer uma das abordagens. Baker (2003) defende, no entanto, que o treino diversificado nas fases iniciais de desenvolvimento pode ser uma forma de conduzir a elevados níveis de desempenho e sustenta que os treinadores e os cientistas do desporto deveriam considerar a abordagem da diversificação na prática desportiva dos mais jovens como uma alternativa à especialização precoce. A diversificação é apontada por vários autores como apresentando vantagens quer para o desenvolvimento físico, quer para o desenvolvimento psicossocial dos jovens desportistas (Baker \& Côté, 2006, p. 98).

Não obstante, apesar de praticarem uma atividade multidesportos, os triatletas parecem não estar protegidos contra o risco de lesões já que, em comparação com os atletas de natação, ciclismo e atletismo, apresentam a segunda maior taxa de incidência de lesões por sobrecarga do SME (Jacques, 2011). Além disso, embora um estudo em que foram entrevistados 28 triatletas suporte a abordagem da diversificação precoce e não sustente a necessidade de treino especializado na fase inicial de desenvolvimento preconizada pela teoria da prática deliberada, os seus autores reconhecem a necessidade de mais estudos de investigação que permitam compreender os fatores subjacentes à aquisição de elevados níveis de desempenho (Baker et al., 2005).

Côté et al. (2009) defendem ainda que a prática desportiva diversificada, ao contrário da especialização e da prática deliberada de um único desporto podem promover a participação continuada e permitir alcançar o máximo desempenho desportivo. A diversificação precoce parece estar, igualmente, associada a uma maior duração da carreira desportiva dos atletas (Côté et al., 2009). Por outro lado, os resultados de um estudo Dinamarquês revelaram que os atletas de topo se especializam mais tarde, treinam menos na infância e intensificam os seus regimes de treino no final da adolescência (Moesch, Elbe, Hauge \& Wikman, 2011).

Embora Ericsson et al. (1993) defendam que é quase impossível que aqueles que começam mais tarde consigam ultrapassar a vantagem inicial dos que iniciam a prática deliberada numa idade precoce, acumulando desse modo um grande número de horas de prática deliberada ao longo do tempo (Côté et al., 2009), 
e apesar de diversos estudos mostrarem uma relação positiva entre o tempo despendido na prática desportiva e o elevado desempenho dos desportistas, Côté et al. (2009) defendem que a participação em diversas atividades desportivas permite que as crianças experimentem uma série de "diferentes ambientes físicos, cognitivos, afetivos e psicossociais" e que as diversas capacidades adquiridas através da participação em vários desportos e em atividades lúdicas durante a infância proporcionam às crianças as capacidades físicas, pessoais e mentais necessárias para se especializarem numa atividade desportiva durante a adolescência.

A especialização precoce é muitas vezes responsabilidade dos pais e coloca uma enorme pressão sobre as crianças e adolescentes que pode conduzir ao isolamento social, ao "burnout" e a lesões por sobre esforço (Brenner \& Council on Sports Medicine Fitness, 2007; Malina, 2010), pondo em risco o seu bem-estar físico, mental e social. Essa opção pode, ainda, ser influenciada pelos professores ou treinadores, ou resultar da vontade da própria criança, para além de outras influências externas como os meios de comunicação social, a indústria de bens e serviços desportivos, os dirigentes desportivos e até mesmo a política internacional (Malina, 2010).

\section{O treino excessivo}

A Comissão Europeia considera que o desporto favorece a saúde, exceto quando associado a treino excessivo (European Comission, 2009). Embora não exista uma medida padrão que permita avaliar o treino excessivo, a sobrecarga física associada a longos períodos de treino intenso é considerada um fator de risco para a saúde dos jovens atletas (Cook \& Leit, 1995; European Comission, 2009; Helms, 1997), podendo originar lesões permanentes devido à imaturidade do SME (Anderson, 2005; Bernhardt \& Landry, 1995; Dalton, 1992; Gerrard, 1993). De acordo com Micheli \& Nielson (2008), “é importante ter em conta que a resposta do esqueleto em desenvolvimento ao treino repetitivo é bastante diferente do de um esqueleto adulto". Diversos autores defendem que o treino excessivo sem o tempo necessário para recuperar da fadiga entre sessões de treino e competições é um fator de risco das lesões desportivas dos jovens atletas (Cosca \& Navazio, 2007; Ivkovic et al., 2007; Luke et al., 2010). Por outro lado, o facto de muitas crianças participarem em desportos durante todo o ano e, por vezes, em várias equipas simultaneamente (Brenner \& Council on Sports Medicine Fitness, 2007), aumenta o risco de lesões por sobrecarga do SME.

\section{Fatores individuais}

A idade e o género, assim como a condição física e mental da criança ou do adolescente são fatores a ter em conta no planeamento da sua atividade desportiva. Mesmo entre atletas da mesma idade há que atender às diferenças biológicas de cada um deles, nomeadamente o estado de desenvolvimento do SME, que não dependem apenas da sua idade cronológica (Helms, 1997) e que podem estar associadas a fatores genéticos, ao metabolismo, ao estado de saúde, a fatores socioeconómicos ou a fatores culturais, entre outros. A imaturidade do SME é um dos fatores intrínsecos que contribui para um maior risco neste grupo populacional (Adirim \& Cheng, 2003; Vandervliet et al., 2007). No entanto, para além das especificidades do próprio crescimento das crianças e adolescentes, os aspetos psicológicos como o nível de maturidade e a autoestima desempenham também um importante papel (DiFiori, 1999) e, conforme Malina (2010) refere, "os jovens desportistas, incluindo os atletas talentosos, são crianças e adolescentes com as necessidades próprias das crianças e adolescentes".

\section{Consequências de longo prazo}

Muitos são os autores que abordam a questão dos eventuais impactos que as lesões sofridas pelas crianças e adolescentes durante 
a prática de atividades físicas e desportivas podem ter, a longo prazo, na saúde do SME (Helms, 1997; Maffulli, Longo, Gougoulias, Loppini, et al., 2010). Muitos defendem que as crianças que praticam regimes intensivos de treino e participam em competições frequentes podem vir a sentir, na idade adulta, as consequências de longo prazo das lesões sofridas (Helms, 1997; Maffulli, Longo, Gougoulias, Caine, et al., 2010). Embora muitas das lesões desportivas dos jovens atletas possam ser ultrapassadas com tratamento e repouso, o treino excessivo e as lesões dos jovens atletas podem resultar no desenvolvimento de patologias da coluna e de lombalgias durante o seu crescimento (Maffulli, Longo, Gougoulias, Loppini, et al., 2010). Um estudo de revisão mostra que o treino desportivo intenso e prolongado pode provocar alterações patológicas no SME que, em casos extremos, pode conduzir a perturbações do crescimento e, a longo prazo, resultar em significativa incapacidade, embora os seus autores reconheçam não existirem evidências suficientes que permitam concluir se os benefícios da participação em desportos excedem os riscos incorridos (Maffulli, Longo, Gougoulias, Caine, et al., 2010).

\section{Questões Éticas}

Conhecidos que são os efeitos da sobrecarga do SME em desenvolvimento de crianças e adolescentes, embora não sendo possível avaliar com exatidão o impacto de longo prazo para a sua saúde, sujeitá-los a longos períodos de treino intenso constitui um grave problema ético. Além disso, "aos jovens atletas que se especializam num único desporto podem estar a ser negados os benefícios da atividade diversificada enquanto enfrentam as exigências físicas, fisiológicas e psicológicas adicionais resultantes do treino intenso e da competição" (Anderson et al., 2000). Vários estudos concluem que as crianças envolvidas na prática de desportos deveriam ser encorajadas a participar numa variedade diversificada de atividades e a desenvolver uma vasta gama de capacidades (Anderson et al., 2000; World Health Organization, 2010, p. 18).

A situação é tanto mais grave quando são, muitas vezes, os próprios pais a estimular práticas desportivas pouco adequadas às necessidades físicas, psicológicas e sociais dos seus filhos. Diversos autores reconhecem que o treino excessivo de crianças e adolescentes é muitas vezes resultado da pressão dos pais (Brenner \& Council on Sports Medicine Fitness, 2007; Silva, Fernandes \& Celani, 2001) e dos dirigentes, no que respeita à obtenção de resultados (Silva et al., 2001). Conforme Anderson et al. (2000) defendem, as expectativas irrealistas por parte dos pais e/ou a exploração dos jovens atletas para obter ganhos podem, além dos efeitos físicos, contribuir para consequências psicológicas negativas para os jovens atletas de "elite". Por outro lado, a ansiedade e o stress podem afetar as crianças envolvidas em modalidades desportivas de elevada carga competitiva e por em causa o seu bem-estar físico e emocional (Anderson et al., 2000). Também a American Academy of Pediatrics reconhece os efeitos negativos da prática desportiva quando as exigências e expectativas excedem a maturidade e a preparação dos participantes (Committee on Sports Medicine Fitness and Committee on School Health, 2001).

No Livro Branco sobre o Desporto, a Comissão Europeia admite a existência de más práticas nas atividades de alguns agentes desportivos (por exemplo, a exploração de jogadores menores de idade) e reconhece a necessidade imperiosa de proteger a saúde e a segurança dos jogadores, particularmente dos menores, e combater as atividades criminosas que prejudicam o desporto (Commission of the European Communities, 2007).

Cabe sobretudo aos pais, mas também aos professores e treinadores, proporcionar às crianças e adolescentes a participação numa multiplicidade de atividades físicas 
(World Health Organization, 2010, p. 18) que promovam o seu normal e saudável desenvolvimento físico, mental e social.

\section{Prevenção}

As estratégias de promoção das atividades físicas e desportivas adotadas a nível mundial (World Health Organization 2010, Commission of the European Communities 2007, Bone and Joint Decade 2000-2010) irão certamente levar cada vez mais crianças e adolescentes a praticar essas atividades pelo que a prevenção assume uma importância fundamental no sentido de evitar as graves consequências individuais e sociais. De modo a reduzir o enorme impacto na qualidade de vida dos indivíduos e as consequências socioeconómicas para a sociedade relacionadas com as doenças músculo-esqueléticas, as estratégias da European Bone and Joint Decade 2004 para a globalidade da população recomendam que as pessoas de todas as idades adotem um estilo de vida saudável e evitem riscos específicos relacionados com a saúde músculo-esquelética, aconselhando a "promoção da saúde nos locais (...) relacionados com as atividades desportivas para evitar o uso anormal e excessivo do sistema músculo-esquelético" (European Bone and Joint Health Strategies Project, 2004, p. 6). Estas recomendações incluem: atividade física regular, a manutenção de um peso corporal saudável, uma dieta equilibrada, a abstenção de fumar, a promoção de programas de prevenção de acidentes para evitar as LME, a promoção da saúde relacionada com as atividades desportivas de modo a evitar a sobrecarga do SME e uma grande consciencialização pública e individual dos problemas relacionados com o SME (European Bone and Joint Health Strategies Project, 2004).

O American College of Sports Medicine (1993) defende que até $50 \%$ do total das lesões desportivas das crianças e adolescentes, envolvidos em desportos organizados, podem ser prevenidas. Similarmente, outros autores defendem que muitas das lesões recreativas (Purvis \& Burke, 2001) e desportivas por sobrecarga (Demorest \& Landry, 2003) podem ser prevenidas.

\section{Exame de pré-participação, vigilância médica e} supervisão

De modo a salvaguardar o direito de todas as crianças e adolescentes a um crescimento saudável e equilibrado, o início de qualquer modalidade desportiva deve ser precedido de um exame médico, que deve ser repetido periodicamente (Bratton, 1997; Greydanus \& Patel, 2008; Shaffer, 1983). O exame médico prévio ou de pré-participação é utilizado para a identificação de problemas médicos que possam afetar a participação segura e eficaz em atividades desportivas organizadas (Carek \& Hunter, 2001; Rooks \& Micheli, 1988) e prevenir futuras lesões (Hergenroeder, 1997; Rowland, 1986). O American College of Sports Medicine (1993) considera que as estratégias de prevenção devem ser tidas em conta durante a realização do exame que precede o início da prática desportiva das crianças e adolescentes. Outros autores reconhecem a importância do exame médico antes de as crianças e adolescentes iniciarem a prática de atividades desportivas (Fuller, Ojelade \& Taylor, 2007; Myers \& Sickles, 1998), incluindo aquelas que apresentam algum tipo de incapacidade (Ramirez et al., 2009).

Este procedimento é fundamental uma vez que o SME das crianças e adolescentes se encontra ainda em desenvolvimento e, além do mais, permite adequar os níveis de atividade física às capacidades individuais de cada criança ou adolescente.

A American Academy of Pediatrics considera que deve caber aos pediatras determinar quando a criança está pronta para participar em desportos organizados (Committee on Sports Medicine Fitness and Committee on School Health, 2001).

Por seu lado, Stanitski defende que os orto- 
pedistas deveriam tomar parte ativa no aconselhamento dos pacientes sobre o equipamento e os programas de treino para prevenir abusos por parte dos pais e dos treinadores que possam conduzir a incapacidades músculo-esqueléticas a longo prazo (Stanitski, 1988).

Apesar da importância do exame médico antes do início da prática desportiva ser indiscutível, alguns autores consideram que ele não é adequadamente (Wingfield, Matheson \& Meeuwisse, 2004) ou uniformemente implementado (Mick \& Dimeff, 2004; Wingfield et al., 2004), enquanto outros entendem que eles devem ser realizados por equipas médicas multidisciplinares (medicina geral, pediatria, ortopedia, ...) (Joy, Paisley, Price, Rassner \& Thiese, 2004) e outros especialistas (Armsey \& Hosey, 2004). A participação crescente do pessoal de enfermagem no exame de pré-participação é igualmente referida (Gemberling, 1996).

Vários estudos consideram que o SME é um dos aspetos que deve ser observado no exame médico de pré-participação (Fields, 1994; Fields \& Delaney, 1990; Fuller et al., 2007; Hergenroeder, 1997; Joy et al., 2004; Mick \& Dimeff, 2004; Rooks \& Micheli, 1988; Shaffer, 1983), o que parece pertinente se atendermos a que os motivos apontados para a recusa ou a aprovação condicional da participação em atividades desportivas dos jovens atletas incluem, entre outros aspetos, os problemas músculo-esqueléticos (Magnes, Henderson \& Hunter, 1992; Mick \& Dimeff, 2004; Rifat, Ruffin \& Gorenflo, 1995).

Para além do exame médico de pré-participação, a vigilância médica regular dos jovens desportistas assume uma importância fundamental na verificação do seu estado geral de saúde e para assegurar o seu crescimento e desenvolvimento normais.

Por seu lado, a supervisão da prática desportiva pelos pais e pelos professores e treinadores com formação adequada, que tenha em conta a opinião dos médicos, desempenha um papel essencial na prevenção das lesões desportivas
(Cook \& Leit, 1995) e na promoção da saúde das crianças e adolescentes. Em particular, a supervisão dos pais pode desempenhar um papel fundamental na prevenção de lesões por sobrecarga, ao adiar a especialização numa única modalidade desportiva para o final da fase de crescimento do SME dos seus filhos e ao evitar que ela ocorra antes da adolescência (American College of Sports Medicine, 1993; DiFiori, 2010).

\section{Programas de treino interdisciplinares e multi-} intervenção

Os programas de treino desempenham um papel essencial na prevenção de lesões. Para proporcionarem aos jovens desportistas um crescimento físico, mental e social equilibrado e saudável e evitar as consequências de longo prazo das lesões, os programas de treino devem ser elaborados em colaboração com os próprios atletas, os pais, médicos (pediatras, médicos de clínica geral, ortopedistas), psicólogos, professores/treinadores e ter em conta a sua imaturidade física e psicológica (Shanmugam \& Maffulli, 2008). Diversos autores consideram que cabe aos pais, treinadores e profissionais de saúde tentar minimizar o potencial das atividades desportivas para causar lesões e incapacidade e permitir às crianças e adolescentes gozarem todos os seus benefícios (Bruns \& Maffulli, 2000; Dalton, 1992; Davis, 2004). Davis (2004) considera ainda que idêntica responsabilidade lhes deve ser atribuída no caso das atividades recreativas, para além de garantir a saúde de longo prazo das crianças.

Por outro lado, os programas de treino devem incluir, simultaneamente, diferentes estratégias de prevenção. Um estudo de revisão mostra que os 6 programas de treino multi-intervenção analisados, envolvendo 2809 participantes, foram eficazes, tendo contribuído para uma redução do risco igual ou superior a 50\%, em 5 deles (Aaltonen et al., 2007). 
Conjunto diversificado de atividades e repouso adequado

A OMS considera que, "as crianças e jovens devem ser encorajados a participar em atividades físicas variadas ... que sejam agradáveis e seguras" e recomenda, para a faixa etária dos 5 aos 17 anos, atividade física moderada a vigorosa, com a duração diária mínima de 60 minutos, realizada no contexto da família, da escola, e de atividades na comunidade e que deve compreender brincadeira, jogos, desportos, transporte, atividades recreativas, educação física ou exercício planeado (World Health Organization, 2010, pp. 7, 18).

Tendo em conta que a imaturidade do SME das crianças e adolescentes limita a sua capacidade para suportar a intensidade dos treinos que habitualmente a prática de desportos requer, em especial as modalidades desportivas de elevada carga competitiva, muitos autores defendem que lhes deve ser proporcionada a prática de atividade física que abranja diversas modalidades de forma a promover um crescimento equilibrado e saudável (Anderson et al., 2000; Johnson, 2008; World Health Organization, 2010). Devem-lhes, além do mais, ser proporcionados os adequados períodos de repouso entre treinos e/ou competições (Ivkovic et al., 2007; Johnson, 2008).

\section{Equipamento de proteção individual}

Embora considerada como inconclusiva em alguns estudos (Abernethy \& Bleakley, 2007), a eficácia do equipamento adequado na prevenção de lesões desportivas é reconhecida e recomendada por vários autores (Aaltonen et al., 2007; Adirim \& Cheng, 2003) e de utilização generalizada entre os desportistas profissionais, em particular em algumas modalidades desportivas que apresentam riscos específicos como sucede com o ciclismo (Aleman \& Meyers, 2010). A sua utilização deve, igualmente, ser generalizada no caso das atividades recreativas que apresentem riscos especiais, de modo a reduzir o risco de lesões entre os praticantes dessas modalidades.

Formação pré-graduada e pós-graduada dos profissionais de saúde

Muitos autores defendem a necessidade de incluir nos currículos das escolas médicas a temática das LME por considerarem que a preparação teórica e prática dos profissionais de saúde não permite dar uma resposta eficaz às necessidades crescentes. Vários autores consideram a necessidade de melhorar, em particular, o ensino pré-graduado nas escolas médicas sobre as LME (Åkesson, Dreinhofer \& Woolf, 2003; Dequeker, Rasker \& Woolf, 2000) de modo a satisfazer as necessidades crescentes das pessoas que sofrem dessas doenças (Åkesson \& Woolf, 2007). Similarmente, alguns autores alertam para a necessidade de incluir as temáticas da Medicina Desportiva nos currículos dos médicos pediatras (Demorest, Bernhardt, Best \& Landry, 2005).

Em Portugal, o Programa Nacional de Protecção contra as Doenças Reumáticas (PNCDR) do Ministério da Saúde define como estratégias de formação a implementar, a "promoção, junto das Faculdades de Medicina, do aumento do número de horas de formação pré e pós-graduada em reumatologia” (E12), a "promoção da formação obrigatória em reumatologia no Internato Complementar de Medicina Geral e Familiar" (E14) e a "promoção da formação específica, na área do SME e das doenças reumáticas, dos profissionais de saúde não médicos, dos técnicos superiores de desporto, e dos professores dos diversos níveis de ensino" (E15) (Direcção-Geral de Saúde, 2004, p. 10).

\section{Educação e consciencialização}

A educação e a consciencialização da sociedade desempenham um importante papel na prevenção das LME (Demorest \& Landry, 2003). A educação dos pais, dos profissionais de saúde, dos professores e treinadores, mas também dos jovens desportistas, e a conscien- 
cialização da população em geral sobre os riscos inerentes à prática desportiva e as medidas preventivas a adotar no sentido de os minimizar desempenham um papel fundamental para a redução do número e da gravidade das LME e para a promoção de uma vida adulta saudável. Pais e educadores esclarecidos podem adotar as decisões mais corretas para a proteção e a promoção da saúde dos seus filhos e educandos na hora de decidir qual o tipo de atividade física que melhor se adequa ao perfil individual de cada um deles. A Bone and Joint Decade considera que a população que participa em atividades físicas ou desportos apresenta risco de LME e recomenda uma abordagem multidisciplinar na educação dos participantes (European Bone and Joint Health Strategies Project, 2004).

No caso de Portugal, as estratégias de formação do PNCDR, a implementar a nível nacional, regional e local, incluem o "desenvolvimento de parcerias multissectoriais para a divulgação, junto da população geral, de informação genérica sobre as doenças reumáticas e sua prevenção" (E19) (Direcção-Geral de Saúde, 2004, p. 11).

\section{CONCLUSÕES}

Não obstante os incontestáveis benefícios da prática regular de exercício físico para a saúde das crianças e adolescentes, parece existir um número crescente de lesões associadas à sua prática. Deve merecer particular atenção, a possibilidade de um número crescente de crianças se especializarem precocemente numa única modalidade já que o SME ainda em desenvolvimento associado a programas de treino intensivo durante longos períodos de tempo, sem os adequados períodos de repouso para recuperação da fadiga, podem conduzir a lesões irreversíveis, com reflexos para a vida adulta.

Para além dos elevados custos socioeconómicos resultantes das LME em jovens desportistas, a prática de atividades desportivas que possam por em causa o seu crescimento normal e equilibrado levanta questões de natureza ética que devem ser consideradas. De modo a prevenir a ocorrência de lesões que possam por em causa o crescimento normal e equilibrado das crianças e adolescentes devem ser adotadas medidas tais como: (1) exame médico antes do início da atividade desportiva que comprove a capacidade da criança ou adolescente para o exercício da atividade em causa e vigilância médica periódica do seu estado de saúde e desenvolvimento físico e mental; (2) supervisão adequada por parte dos pais, professores e treinadores; (3) programas de treino interdisciplinares e multi-intervenção que tenham em conta o desenvolvimento físico e mental das crianças e adolescentes; (4) educação dos pais, professores/treinadores e das próprias crianças e adolescentes sobre as vantagens da prática regular de exercício físico mas também dos riscos para a sua saúde e das medidas que devem adotar de modo a usufruir plenamente das vantagens que ela proporciona; (5) formação pré-graduada e pós-graduada dos profissionais de saúde que lhes permita fazer face à crescente tendência das LME e, por um lado, proporcionar, aos professores e treinadores, informação clínica relevante que permita a estes profissionais um adequado planeamento da atividade desportiva deste especial grupo populacional e, por outro lado, facultar um aconselhamento eficaz aos pais, crianças e adolescentes; (6) ações públicas de consciencialização de modo a promover a prática regular de atividade física e a escolha das opções mais adequadas à proteção e promoção da saúde que, na faixa etária analisada, deve compreender um conjunto diversificado de atividades físicas e (7) utilização dos equipamentos de proteção adequados à prática de cada modalidade.

\section{Agradecimentos:}

Nada declarado.

Conflito de Interesses:

Nada declarado. 
Financiamento:

Nada declarado.

\section{REFERÊNCIAS}

Aaltonen, S., Karjalainen, H., Heinonen, A., Parkkari, J., \& Kujala, U. M. (2007). Prevention of sports injuries: systematic review of randomized controlled trials. Archives of Internal Medicine, 167(15), 1585-1592.

Abernethy, L., \& Bleakley, C. (2007). Strategies to prevent injury in adolescent sport: a systematic review. British Journal of Sports Medicine, 41 (10), 627-638.

Adirim, T. A., \& Cheng, T. L. (2003). Overview of injuries in the young athlete. Sports medicine (Auckland, NZ), 33(1), 75-81.

Åkesson, K., Dreinhofer, K. E., \& Woolf, A. D. (2003). Improved education in musculoskeletal conditions is necessary for all doctors. Bulletin of the World Health Organization, 81 (9), 677-683.

Åkesson, K., \& Woolf, A. D. (2007). Education in Musculoskeletal Health - How Can It Be Improved to Meet Growing Needs? The Journal of Rheumatology, 34(3), 455-457.

Aleman, K. B., \& Meyers, M. C. (2010). Mountain Biking Injuries in Children and Adolescents. Sports Medicine, 40(1), 77-90.

American Academy of Orthopaedic Surgeons. (2010). Pediatric Sports Injuries: The Silent Epidemic. ScienceDaily Retrieved November 30, 2010, from http://www.sciencedaily.com/ releases/2010/03/100310083441.htm

American College of Sports Medicine. (1993). The prevention of sport injuries of children and adolescents. Med Sci Sports Exerc, 25(8 Suppl), $1-7$.

Anderson, S. J. (2005). Sports Injuries. Disease-a-Month, 51 (8-9), 438-542.

Anderson, S. J., Griesemer, B. A., Johnson, M. D., Martin, T. J., McLain, L. G., Rowland, T. W., et al. (2000). Intensive training and sports specialization in young athletes. Pediatrics, 106(1), 154-157.

Armsey, T. D., \& Hosey, R. G. (2004). Medical aspects of sports: epidemiology of injuries, preparticipation physical examination, and drugs in sports. Clin Sports Med, 23 (2), 255-279, vii.

Baker, J. (2003). Early Specialization in Youth Sport: a requirement for adult expertise? High Ability Studies, 14(1), 85.

Baker, J., Cobley, S., \& Fraser-Thomas, J. (2009). What do we know about early sport specialization? Not much! High Ability Studies, 20(1), 77-89.

Baker, J., \& Côté, J. (2006). Shifting training requirements during athlete development: Deliberate practice, deliberate play and other sport involvement in the acquisition of sport expertise. In D. Hackfort \& G. Tenenbaum (Eds.), Essential Processes for Attaining Peak Performance (Vol. 1, pp. 92-109). Oxford: Meyer \& Meyer Sport (UK) Ltd.

Baker, J., Côté, J., \& Deakin, J. M. (2005). Expertise in Ultra-Endurance Triathletes Early Sport Involvement, Training Structure, and the Theory of Deliberate Practice. Journal of Applied Sport Psychology, 17(1), 64-78.

Belechri, M., Petridou, E., Kedikoglou, S., Trichopoulos, D., \& Sports Injuries' European Union group. (2001). Sports injuries among children in six European Union countries. European Journal of Epidemiology, 17(11), 1005-1012.

Benjamin, M., Kumai, T., Milz, S., Boszczyk, B. M., Boszczyk, A. A., \& Ralphs, J. R. (2002). The skeletal attachment of tendons - tendon 'entheses'. Comparative Biochemistry and Physiology - Part A: Molecular \& Integrative Physiology, 133(4), 931-945.

Bernhardt, D. T., \& Landry, G. L. (1995). Sports injuries in young athletes. Adv Pediatr, 42, 465-500.

Bratton, R. L. (1997). Preparticipation screening of children for sports. Current recommendations. Sports Medicine, 24(5), 300-307.

Brenner, J. S., \& Council on Sports Medicine Fitness. (2007). Overuse Injuries, Overtraining, and Burnout in Child and Adolescent Athletes. Pediatrics, 119(6), 1242-1245.

Bruns, W., \& Maffulli, N. (2000). Lower limb inju- 
ries in children in sports. Clin Sports Med, 19(4), 637-662.

Buckley, S. L. (1994). Sports injuries in children. Current Opinion in Pediatrics, 6(1), 80-84.

Caine, D., DiFiori, J. P., \& Maffulli, N. (2006). Physeal injuries in children's and youth sports: reasons for concern? British Journal of Sports Medicine, 40(9), 749-760.

Callender, S. S. (2010). The Early Specialization of Youth in Sports. Athletic Training \& Sports Health Care: The Journal for the Practicing Clinician, 2(6), 255-257.

Carek, P. J., \& Hunter, L. (2001). The preparticipation physical examination for athletics: a critical review of current recommendations. The Lebanese medical journal, 49(5), 292-297.

Carty, H. (1998). Children's sports injuries. European Journal of Radiology, 26(2), 163-176.

CDC. (n.d., 2009, July 27). Sports Injuries: The Reality. Retrieved October 7, 2011, from http://www.cdc.gov/safechild/Sports_Injuries/

Cirak, B., Ziegfeld, S., Knight, V. M., Chang, D., Avellino, A. M., \& Paidas, C. N. (2004). Spinal injuries in children. Journal of Pediatric Surgery, $39(4), 607-612$.

Colvin, A. C., \& Lynn, A. (2010). Sports-Related Injuries in the Young Female Athlete. Mount Sinai Journal of Medicine, 77(3), 307-314.

Commission of the European Communities. (2007). White Paper on Sport., $\operatorname{COM}(2007) 391$ final. (Vol. 2010. Available from http://ec.europa.eu/ sport/white-paper/index_en.htm

Committee on Sports Medicine Fitness and Committee on School Health. (2001). Organized Sports for Children and Preadolescents. Pediatrics, 107(6), 1459-1462.

Conn, J. M., Annest, J. L., Gilchrist, J., \& Ryan, G. W. (2004). Injuries from paintball game related activities in the United States, 1997-2001. Injury Prevention, 10(3), 139-143.

Cook, P. C., \& Leit, M. E. (1995). Issues in the pediatric athlete. The Orthopedic clinics of North America, 26(3), 453-464.

Cosca, D. D., \& Navazio, F. (2007). Common problems in endurance athletes. American Family
Physician, 76(2), 237-244.

Côté, J., Lidor, R., \& Hackfort, D. (2009). ISSP position stand: To sample or to specialize? Seven postulates about youth sport activities that lead to continued participation and elite performance. International Journal of Sport and Exercise Psychology, 7(1), 7-17.

Dalton, S. E. (1992). Overuse injuries in adolescent athletes. Sports medicine, 13(1), 58-70.

Damore, D. T., Metzl, J. D., Ramundo, M., Pan, S., \& van Amerongen, T. (2003). Patterns in childhood sports injury. Pediatric Emergency Care, 19(2), 65-67.

Davis, E. K. (2004). Sports and recreational injuries in children and adolescents: prevention and education. J Okla State Med Assoc, 97(1), 18-21.

Demorest, R. A., Bernhardt, D. T., Best, T. M., \& Landry, G. L. (2005). Pediatric residency education: Is sports medicine getting its fair share? Pediatrics, 115(1), 28-33.

Demorest, R. A., \& Landry, G. L. (2003). Prevention of pediatric sports injuries. Curr Sports Med Rep, 2(6), 337-343.

Dequeker, J., Rasker, J. J., \& Woolf, A. D. (2000). Educational issues in rheumatology. Best Practice E Research Clinical Rheumatology, 14(4), 715-729.

DiFiori, J. P. (1999). Overuse Injuries in Children and Adolescents. The Physician and Sports Medicine, 27(1), 75-89.

DiFiori, J. P. (2010). Evaluation of Overuse Injuries in Children and Adolescents. Current Sports Medicine Reports, 9(6), 372-378.

Direcção-Geral de Saúde. (2004). Programa Nacional Contra as Doenças Reumáticas - Despacho Ministerial de 26-03-2004. Available from http://www.dgs.pt/upload/membro.id/ ficheiros/i006345.pdf

Dohin, B. (2010). Sport overuse injuries of growth plate and apophysis in children. Archives De Pediatrie, 17(6), 616-617.

European Bone and Joint Health Strategies Project. (2004). European action towards better musculoskeletal health: A Public Health Strategy to Reduce the Burden of Musculoskeletal Conditions: The Bone \& Joint Decade. 
European Comission. (2009). Health. Retrieved December 13, 2010, from http://ec.europa.eu/ sport/what-we-do/doc27_en.htm

European Commission. (2009). Education and training. Retrieved December 13, 2010, from http://ec.europa.eu/sport/what-we-do/doc31_ en.htm

European Foundation for the Improvement of Living and Working Conditions. (2007). Managing musculoskeletal disorders: European Foundation for the Improvement of Living and Working Conditions.

Fields, K. B. (1994). Clearing athletes for participation in sports. The North Carolina Medical Society Sports Medicine Committee's recommended examination. North Carolina Medical Journal, 55(4), 116-121.

Fields, K. B., \& Delaney, M. (1990). Focusing the Preparticipation Sports Examination. Journal of Family Practice, 30(3), 304-310.

FIMS/WHO Ad Hoc Committee on Sports and Children. (1998). Sports and children: consensus statement on organized sports for children. FIMS/WHO ad Hoc Committee on Sports and Children. Bulletin of the World Health Organization, 76(5), 445-447.

Foster, H. E., \& Cabral, D. A. (2006). Is musculoskeletal history and examination so different in paediatrics? Best Practice \& Research Clinical Rheumatology, 20(2), 241-262.

Fuller, C. W., Ojelade, E. O., \& Taylor, A. (2007). Preparticipation medical evaluation in professional sport in the UK: theory or practice? British Journal of Sports Medicine, 41 (12), 890-896.

Gemberling, C. (1996). Preparticipation sports evaluation: An overview. Nurse Practitioner Forum-Current Topics and Communications, 7(3), 125-135.

Gerrard, D. F. (1993). Overuse injury and growing bones: the young athlete at risk. Br J Sports Med, 27(1), 14-18.

Goldberg, A. S., Moroz, L., Smith, A., \& Ganley, T. (2007). Injury surveillance in young athletes A clinician's guide to sports injury literature. Sports Medicine, 37(3), 265-278.
Gottschalk, A. W., \& Andrish, J. T. (2011). Epidemiology of Sports Injury in Pediatric Athletes. Sports Medicine and Arthroscopy Review, 19(1), 2-6. Greydanus, D. E., \& Patel, D. R. (2008). Sports preparticipation examination and chronic illness: Let the games begin! International Journal on Disability and Human Development, 7(3), 291-295.

Grimmer, K., \& Williams, J. (2003). Injury in junior Australian Rules footballers. Journal of Science and Medicine in Sport, 6(3), 328-338.

Hawkins, D., \& Metheny, J. (2001). Overuse injuries in youth sports: biomechanical considerations. Medicine and Science in Sports and Exercise, 33(10), 1701-1707.

Helms, P. J. (1997). Sports injuries in children: should we be concerned? Archives of Disease in Childhood, 77(2), 161-163.

Hergenroeder, A. C. (1997). The preparticipation sports examination. Pediatric Clinics of North America, 44(6), 1525-1540.

Hutchinson, M. R., \& Nasser, R. (2000). Common Sports Injuries in Children and Adolescents. Medscape General Medicine, 2(4). Retrieved from http://www.medscape.com/viewarticle/408524 Ivkovic, A., Franic, M., Bojanic, I., \& Pecina, M. (2007). Overuse injuries in female athletes. Croatian medical journal, 48(6), 767-778.

Jacques, R. (2011). Triathlon injuries. In M. Hutson \& C. Speed (Eds.), Sports Injuries (pp. 467-470). Oxford: Oxford University Press.

Johnson, J. H. (2008). Overuse Injuries in Young Athletes: Cause and Prevention. Strength and Conditioning Journal, 30(2), 27-31.

Joy, E. A., Paisley, T. S., Price, R., Rassner, L., \& Thiese, S. M. (2004). Optimizing the collegiate preparticipation physical evaluation. Clinical Journal of Sport Medicine, 14(3), 183-187.

Kerssemakers, S. P., Fotiadou, A. N., de Jonge, M. C., Karantanas, A. H., \& Maas, M. (2009). Sport injuries in the paediatric and adolescent patient: a growing problem. Pediatric Radiology, 39(5), 471-484.

Lord, J., \& Winell, J. J. (2004). Overuse injuries in pediatric athletes. Current Opinion in Pediatrics, 
16(1), 47-50.

Luke, A., Lazaro, R. M., Bergeron, M. F., Keyser, L., Benjamin, H., Brenner, J., et al. (2010). Fatigue is Perceived as a Risk Factor in Overscheduling Injuries in Young Athletes. Clinical Journal of Sport Medicine, 20(3), 228-235.

Maffulli, N., \& Baxter-Jones, A. D. (1995). Common skeletal injuries in young athletes. Sports medicine (Auckland, N Z), 19(2), 137-149.

Maffulli, N., \& Bruns, W. (2000). Injuries in young athletes. European Journal of Pediatrics, 159(1-2), 59-63.

Maffulli, N., Longo, U. G., Gougoulias, N., Caine, D., \& Denaro, V. (2010). Sport injuries: a review of outcomes. British Medical Bulletin, 97(1), 47-80.

Maffulli, N., Longo, U. G., Gougoulias, N., Loppini, M., \& Denaro, V. (2010). Long-term Health Outcomes of Youth Sports Injuries. British Journal of Sports Medicine, 44, 21-25.

Maffulli, N., Longo, U. G., Spiezia, F., \& Denaro, V. (2010). Sports injuries in young athletes: long-term outcome and prevention strategies. Phys Sportsmed, 38(2), 29-34.

Magnes, S. A., Henderson, J. M., \& Hunter, S. C. (1992). What Conditions Limit Sports Participation - Experience with 10540 Athletes. Physician and Sportsmedicine, 20(5), 143-160.

Malina, R. M. (2009). Children and Adolescents in the Sport Culture: The Overwhelming Majority to the Select Few. Journal of Exercise Science $\mathcal{E}$ Fitness, 7(2, Supplement 1), S1-S10.

Malina, R. M. (2010). Early Sport Specialization: Roots, Effectiveness, Risks. Current Sports Medicine Reports, 9(6), 364-371.

Metzl, J. D. (1999). Sports-specific concerns in the young athlete: Soccer. Pediatric Emergency Care, 15(2), 130-134.

Micheli, L. J., Glassman, R., \& Klein, M. (2010). The prevention of sports injuries in children. Clinics in Sports Medicine, 19(4), 821-834.

Micheli, L. J., \& Klein, J. D. (1991). Sports injuries in children and adolescents. Br J Sports Med, 25(1), 6-9.

Micheli, L. J., \& Nielson, J. H. (2008). Overuse Injuries in the Young Athlete: Stress Fractures. In H.
Hebestreit \& O. Bar-Or (Eds.), The Young Athlete: Encyclopaedia of Sports Medicine (pp. 151-163): Blackwell Publishing.

Mick, T. M., \& Dimeff, R. J. (2004). What kind of physical examination does a young athlete need before participating in sports? Cleveland Clinic Journal of Medicine, 71 (7), 587-597.

Moesch, K., Elbe, A. M., Hauge, M. L. T., \& Wikman, J. M. (2011). Late specialization: the key to success in centimeters, grams, or seconds (cgs) sports. Scandinavian Journal of Medicine \& Science in Sports.

Möller, A., Åström, M., \& Westlin, N. E. (1996). Increasing incidence of Achilles tendon rupture. Acta Orthopaedica, 67(5), 479-481.

Myers, A., \& Sickles, T. (1998). Preparticipation sports examination. Primary Care, 25(1), 225-236.

O’Rourke, K. P., Quinn, F., Mun, S., Browne, M., Sheehan, J., Cusack, S., et al. (2007). A comparison of paediatric soccer, gaelic football and rugby injuries presenting to an emergency department in Ireland. Injury-International Journal of the Care of the Injured, 38(1), 104-111.

O'Connor, P. J., \& Groves, C. (2005). Trauma and Sports-related Injuries. In D. J. Wilson (Ed.), Paediatric musculoskeletal disease: with an emphasis on ultrasound (pp. 19-38). Oxford, UK: Springer.

Patel, D. R., \& Nelson, T. L. (2000). Sports injuries in adolescents. Med Clin North Am, 84(4), 983-1007, viii.

Purnell, M., Shirley, D., Nicholson, L., \& Adams, R. (2010). Acrobatic gymnastics injury: Occurrence, site and training risk factors. Physical Therapy in Sport, 11 (2), 40-46.

Purvis, J. M., \& Burke, R. G. (2001). Recreational injuries in children: incidence and prevention. The Journal of the American Academy of Orthopaedic Surgeons, 9(6), 365-374.

Ramirez, M., Yang, J. Z., Bourque, L., Javien, J., Kashani, S., Limbos, M. A., et al. (2009). Sports Injuries to High School Athletes With Disabilities. Pediatrics, 123(2), 690-696.

Rifat, S. F., Ruffin, M. T., \& Gorenflo, D. W. (1995). Disqualifying criteria in a preparticipation 
sports evaluation. Journal of Family Practice, 41(1), 42-50.

Rooks, D. S., \& Micheli, L. J. (1988). Musculoskeletal assessment and training: the young athlete. Clinics in Sports Medicine, 7(3), 641-677.

Rowland, T. W. (1986). Preparticipation sports examination of the child and adolescent athlete: changing views of an old ritual. Pediatrician, 13(1), 3-9.

Shaffer, T. E. (1983). The physician's role in sports medicine. Serving the athlete, school, and team. J Adolesc Health Care, 3(4), 227-230.

Shanmugam, C., \& Maffulli, N. (2008). Sports injuries in children. British Medical Bulletin, 86(1), 33-57.

Shields, B. J., \& Smith, G. A. (2006). Cheerleading-related injuries to children 5 to 18 years of age: United States, 1990-2002. Pediatrics, 117(1), 122-129.

Silva, F. M., Fernandes, L., \& Celani, F. O. (2001). Desporto de crianças e jovens - um estudo sobre as idades de iniciação. Revista Portuguesa de Ciências do Desporto, 1(2), 45-55.

Singh, S., Smith, G. A., Fields, S. K., \& McKenzie, L. B. (2008). Gymnastics-related injuries to children treated in emergency departments in the United States, 1990-2005. Pediatrics, 121(4), e954-960.

Stanitski, C. L. (1988). Management of sports injuries in children and adolescents. Orthop Clin North Am, 19(4), 689-698.

Stanitski, C. L. (1997). Pediatric and adolescent sports injuries. Clin Sports Med, 16(4), 613-633.
University of Michigan. (2009). Why prevention: Youth Sports Injury Prevention. Bone \& Joint Injury Prevention \& Rehabilitation Center - University of Michigan. Retrieved November 3, 2010, from http://bjiprc.umich.edu/why.youth.asp

Vandervliet, E. J. M., Vanhoenacker, F. M., Snoeckx, A., Gielen, J. L., Van Dyck, P., \& Parizel, P. M. (2007). Sports-related acute and chronic avulsion injuries in children and adolescents with special emphasis on tennis. British Journal of Sports Medicine, 41(11), 827-831.

Wall, M., \& Côté, J. (2007). Developmental activities that lead to dropout and investment in sport. Physical Education \& Sport Pedagogy, 12(1), 77-87.

Watkins, J., \& Peabody, P. (1996). Sports injuries in children and adolescents treated at a sports injury clinic. Journal of Sports Medicine and Physical Fitness, 36(1), 43-48.

Wiersma, L. D. (2000). Risks and benefits of youth sport specialization: perspectives and recommendations. Pediatric Exercise Science, 12(1), 13-22.

Wingfield, K., Matheson, G. O., \& Meeuwisse, W. H. (2004). Preparticipation evaluation: an evidence-based review. Clinical journal of sport medicine: official journal of the Canadian Academy of Sport Medicine, 14(3), 109-122.

World Health Organization. (2010). Global recommendations on physical activity for health. Geneva: World Health Organization.

(cc) EY-No Todo o conteúdo da revista Motricidade está licenciado sob a Creative Commons, exceto quando especificado em contrário e nos conteúdos retirados de outras fontes bibliográficas. 